\title{
«Un tas de pierres n'a jamais fait une maison»
}

\author{
Ariane Treu, Maurice Hurni, \\ Georges Gabris, \\ Lito Panayotopoulos, \\ Nick Miller, Alain Porchet
}

Comité du Groupement des psychiatres-psychothérapeutes membres de la Société Vaudoise de Médecine GPPV

\section{Correspondance:}

Dr Ariane Treu

Spécialiste en psychiatrie et psychothérapie FMH Spécialiste en psychiatrie et psychothérapie d'enfants et d'adolescents FMH Vice-présidente du GPPV Rue Beau-Séjour 7 CH-1003 Lausanne a.treu@bluewin.ch
Cette phrase du mathématicien Henri Poincaré vient à l'esprit après la lecture du questionnaire reçu par les psychiatres pour la déclaration des démarches qualité dans le cadre du projet «Monitorage de la qualité» de la FMH.

L'idée de cette première étape est la saisie des activités qualité des psychiatres afin de les rendre visibles statistiquement. Ces données seront analysées en termes de coût et d'utilité. Puis les médecins seront évalués sur la base de leurs activités en matière de qualité, les données seront publiées afin de comparer les médecins entre eux et d'instaurer une concurrence en matière de qualité et de prix [1].

L'ambiguïté du projet est de plus en plus évidente.

\section{Les conditions actuelles d'exercice de la médecine psychiatrique sont encadrées et contrôlées de multiples manières}

Au départ, il s'agissait selon la FMH de «rendre plus visible le travail du corps médical en vue de garantir la qualité des soins» [1]. Insidieusement cela se transforme, au nom d'une exigence de transparence, en mise sur pied d'un dispositif de gestion et de contrôle statistique reposant sur des critères formels de productivité, introduisant la norme managériale et l'idée de concurrence du marché dans notre pratique médicale qui jusque-là a pu en être préservée.

En effet, les conditions actuelles d'exercice de la médecine psychiatrique sont encadrées et contrôlées de multiples manières: après l'obtention du diplôme de médecin, il faut au minimum 5 ans de spécialisation pour obtenir le titre FMH en psychiatrie et psychothérapie. Puis la formation continue obligatoire impose 240 heures de formation en 3 ans qui est attestée formellement par un diplôme de formation continue tous les 3 ans. Une partie importante de cette formation est constituée d'un contrôle de la qualité immédiat par les supervisions et intervisions régulières.

Dans ses traitements, le psychiatre doit régulièrement rendre des comptes aux médecins-conseil des assurances pour justifier l'adéquation de ses indications thérapeutiques et de ses coûts. Il est soumis au contrôle de l'économicité de ses traitements par Santé- suisse (indice ANOVA). Les patients mécontents peuvent se plaindre à la Commission de déontologie de la Société cantonale de médecine ou en justice. Ces Sociétés médicales cantonales et leurs groupements de spécialistes sont aussi des éléments de contrôle de l'éthique et de la qualité des traitements. Il faut peutêtre le dire pour le rendre plus visible et ne pas disqualifier toutes ces mesures existantes. Allons-nous demander à un conducteur qui a réussi son permis de conduire, qui suit des cours de perfectionnement réguliers validés formellement tous les trois ans par un diplôme, qui n'a eu aucun problème, que les autres conducteurs estiment, que les passagers ont choisi librement, de justifier de façon transparente pourquoi il conduit bien? L'absurdité de la démarche est évidente.

Mais la lecture du questionnaire «qualité» réserve d'autres surprises. Outre un nouveau recensement des activités de formation continue (rubriques A et C), totalement inutile puisque déjà existant, les autres points relèvent d'une vision gestionnaire de l'activité clinique: vérification de la sécurité et de la gestion des urgences, des concepts de triage, formalisation des aspects relationnels par des enquêtes d'opinion, contrôles, système d'organisation et de gestion, certification du cabinet. Les critères choisis questionnent quant aux valeurs sur lesquelles reposerait cette évaluation.

\section{Gestion, administration et contrôle à la place de pertinence, justesse et intelligibilité}

Selon les recommandations de l'Académie Suisse des Sciences médicales, le relevé, l'analyse et la publication de données concernant la qualité des traitements médicaux devrait respecter 3 critères: pertinence, justesse et intelligibilité. Mesurer suppose un choix préalable de critères pertinents caractérisant la qualité et la transparence devrait exister concernant le choix de ces valeurs et indiquer le responsable de ce choix [2].

Or les paramètres choisis dans ce questionnaire relèvent de valeurs basées prioritairement sur la gestion, l'organisation, le contrôle, l'administration. Certains critères ne sont pas qu'absurdes mais contre-productifs. Ils compromettent la relation thérapeutique principal outil du psychiatre-psychothérapeute - en la réifiant. Si un questionnaire de satisfaction peut avoir un sens pour une prestation hôtelière, l'idée que cela puisse caractériser la qualité du travail psychothérapeutique relève d'une méconnaissance inquiétante de notions centrales telles que la séduction, l'am- 
bivalence, le clivage ou la destructivité. L'acte médical devient une marchandise, le psychiatre un gentil coach bien organisé.

Alors que la médecine clinique a désormais admis l'importance de la complexité, le patient ne se réduisant pas à la maladie, la maladie elle-même étant un processus complexe et donc le soin aussi, de quelle qualité parlons-nous?

La transparence revendiquée dans cette démarche n'a rien à voir avec la vérité, elle désigne l'inconsistance et le vide, la figure d'une médecine formelle vidée de son contenu clinique.

\section{Certains critères ne sont pas qu'absurdes mais}

\section{contre-productifs et compromettent la relation thérapeutique}

d'un tel dispositif d'évaluation de la qualité influencera les pratiques en retour en constituant une nouvelle norme. «Il suffit que l'indicateur d'activité se transforme en norme de performance pour que les médecins finissent par s'ajuster à cette norme au mieux de leurs intérêts: on passe de l'évaluation à la servitude volontaire» [3]. Ainsi il s'agit d'un changement de paradigme du soin psychiatrique qui ne dit pas son nom.

Or dans ce système, la valeur se déduit de la visibilité sociale. On voit bien la catastrophe de ce processus au niveau de la recherche, les chercheurs n'étant plus évalués sur le contenu de leurs recherches, mais sur l'impact factor des revues dans lesquelles ils sont publiés [3]. «Monitorage de la qualité: yes we count!» est intitulé fièrement le dernier éditorial du Dr Daniel Herren dans le BMS [4]. Désormais ce qui compte, c'est ce qui se compte. Peu importe que cela soit établi sur de faux indicateurs, l'essentiel est d'installer au cœur du corps médical une nouvelle manière de concevoir les pratiques professionnelles: produire un tas de pierres standardisées au meilleur coût possible pour être bien coté au palmarès de la qualité. Mais la qualité de quoi au juste? D'un tas de pierres qui n'a jamais fait une maison. conformiste et superficielle, nous pourrons fourni des prestations chiffrées standardisées (transparence que même les assureurs refusent de s'appliquer, pour d'autres raisons) mais nous détruirons cela même qui garantit les conditions du soin: relation privilégiée entre le patient et son médecin, respect de la sphère intime du patient garanti par le secret médical, priorité à la clinique sur la gestion administrative, soins individualisés, prise en compte de la subjectivité, esprit de responsabilité, implication personnelle.

Si nous nous insurgeons contre ce questionnaire, ce n'est pas seulement parce qu'il constitue une collecte de données absurdes pour évaluer la qualité de notre travail, mais c'est surtout parce que l'adoption

\section{Commentaires des SSPP* et SSPPEA** à pro- pos de I'article d'Ariane Treu et al.}

Dans les deux articles du BMS cités par notre collègue Ariane Treu il est question des problèmes et éventuels effets contre-productifs que peuvent avoir les mesures de qualité. Les membres des comités des deux sociétés chargés à la Qualité ainsi que le responsable Qualité de la FMH sont conscients des écueils en lien avec ces démarches. Depuis $\mathbf{1 5}$ ans nous sommes, selon la LAMal, dans I'obligation de démontrer la qualité de nos prestations. Nos efforts ne sont donc pas absurdes ni l'expression d'une servitude.

Société Suisse de Psychiatrie et Psychothérapie

** Société Suisse de Psychiatrie de l'Enfant et de l'Adolescent et Psychothérapie

*** Foederatio Medicorum Psychiatricorum et Psychotherapeuticorum
Si nous ne faisons rien, soit nous aurons à nous contenter de critères d'efficacité, adéquation et économicité (EAE) ou alors d'autres définiront pour nous les critères selon lesquels la qualité de nos prestations sera mesurée.
Le Projet Q-Monitoring n'a pas pour objectif de mesurer la qualité mais de recenser les activités en matière de qualité déjà existantes dans notre pratique et de le communiquer. Cette démarche devrait nous permettre de faire un premier pas dans ce qui semble être une bonne direction. Mme Ariane Treu précise à juste titre les différentes compétences qu'un psychiatre psychothérapeute doit actuellement déjà pouvoir attester. Mais est-ce que d'autres que nous le savent? et qui mieux que nous peut définir pour notre discipline ce qu'est la qualité?

Dr Hans Kurt, Président FMPP***

Dr Yvette Attinger Andreoli, Comité SSPP Ressort Qualité

Dr Hélène Beutler, Comité SSPPEA Ressort Qualité 